\title{
NUMERICAL INVESTIGATION ON SAND EROSION PHENOMENON OF COATED AND UNCOATED VANES IN LOW-PRESSURE GAS TURBINE
}

\author{
YOSHIMASA FUMA ${ }^{1}$, HIROYA MAMORI ${ }^{1}$, NAOYA FUKUSHIMA ${ }^{1}$, \\ MAKOTO YAMAMOTO ${ }^{1} \&$ YOJI OKITA ${ }^{2}$ \\ ${ }^{1}$ Tokyo University of Science, Japan. \\ ${ }^{2} \mathrm{IHI}$ Corporation, Japan.
}

\begin{abstract}
For energy saving and less environmental impact, efficient energy utilization is of importance. In a gasturbine engine, its performance increases as increasing temperature of the turbine inlet flow. However, turbine components are required to be protected from the high temperature flows. Recently, ceramic matrix composite (referred as $\mathrm{CMC}$, hereafter) is expected to be utilized as protecting the gas-turbine components due to the excellent properties of CMC in high temperature conditions: low density, high strength and high rigidity. Therefore, the $\mathrm{CMC}$ allows us to increase the inlet temperature and leads to high performance of gas-turbine engines. On the other hand, sand erosion phenomenon is one of serious problems in gas-turbine engines. Sand particles ingested from the engine inlet impinge and erode the wall surfaces, which can cause engine failure. In order to prevent the sand erosion phenomenon, anti-erosion coatings have been developed and adopted for gas-turbines, although the anti-erosion characteristics of the CMC coating have not completely been clarified. The objective of the present paper is to perform numerical simulations of sand erosion phenomenon on the coated and the uncoated T106 CMC vanes in a low-pressure gas turbine. We investigate the flow field, particle trajectories and the eroded shape of the CMC turbine vanes. The results show that the erosion occurs near the leading edge and at the 90 percent axial chord on the pressure surface in both of the coated and uncoated cases. In the uncoated case, the severe erosion phenomenon is observed especially. Accordingly, we have concluded that the coating obviously played an important role in protecting the CMC vanes from sand erosion.

Keywords: Ceramic Matrix Composites, Eulerian-Lagrangian Approach, Low-Pressure Turbine, Numerical Simulation, Sand Erosion.
\end{abstract}

\section{INTRODUCTION}

For energy saving and less environmental impact, efficient energy utilization is of importance. In gas-turbine engines, when the inlet temperature increases, its performance increases. Recently, ceramic matrix composite (below CMC) is adopted for the gas-turbine components because of the high heat resisting property. The $\mathrm{CMC}$ allows us to increase the inlet temperature and thus it leads to high performance of gas-turbine engines.

On the other hand, sand erosion phenomenon is one of the serious problems in a gas-turbine engine [1], that is, sand particles are ingested from the engine inlet and they impinge on and erode the walls of components. CMC is easily damaged by the phenomenon. Therefore, anti-erosion coatings have been developed and adopted for gas turbines.

The research on sand erosion phenomenon started in Germany in 1930's. Finnie [2] made a theoretical analysis on sand erosion phenomena and proposed an erosion model. However, the model cannot predict the weight loss accurately when the impact angle is large.

Bitter [3, 4] discussed that the sand erosion phenomena consist of deformation wear and cutting wear and then proposed an erosion model. The erosion model sufficiently predicted the erosion phenomena on both ductile and brittle materials for any impinging angle. However, a major drawback of the model is that it is too complicate to apply the model in real situations. 
Thus, Neilson and Gilchrist [5,6] modified the Bitter's model and applied it in practical simulations.

Erosion in gas turbine is affected by many factors such as the ingested particle characteristics, geometry, surface material and operating conditions. Balan and Tabakoff [7] performed an experimental study of the sand erosion phenomena on a single stage axial flow compressor. They found severe erosion phenomena on the leading edge and the pressure side of the rotor blades. They also observed that the erosion is enhanced as increasing of the surface roughness. Suzuki et al. [8] numerically investigated change of the flow field, the particle trajectories, and the eroded wall shape in the compressor, to clarify the effects of sand erosion in the compressor.

In the present paper, we perform numerical simulations of the sand erosion phenomena on a coated and an uncoated T106 CMC vanes in a low-pressure gas turbine. The objective is to reveal robustness of the CMC coating from the sand erosion phenomena. We investigate the flow field, particle trajectories and the eroded shape of the CMC turbine vanes. It is confirmed that the coating on the $\mathrm{CMC}$ vane plays an important role to protect the vane from sand erosion.

\section{NUMERICAL PROCEDURES}

To reproduce the temporal change of the turbine vane surface eroded by sand, we iteratively compute the flow field, the particle trajectories, and the wall deformation that is caused by erosion. Each numerical procedure is described in detail in the following sections.

\subsection{Flow Field}

The three-dimensional and compressible turbulent flow is assumed. The governing equations are the Favre-averaged continuity, Navier-Stokes and energy equations. The modified $k-\varepsilon$ turbulence model [9] is used to estimate turbulence effects. The governing equations are discretized based on the second-order upwind TVD scheme proposed by Yee [10] for the inviscid terms, and the second-order central difference scheme for the viscous terms. The LU-ADI scheme proposed by Fujii and Obayashi [11] is employed for the time integration.

\subsection{Particle Motion}

The particles are captured in a Lagrangian framework in this study. Since the number density of the sand particles in a gas turbine is very small, the one-way coupling is assumed, that is, the particles are affected by the flow whereas the flow is not affected by the particles. Particleparticle collision is ignored because of the low concentration. It is also assumed that all particles are sphere and non-rotating. According to these assumptions, we obtain a simplified $\mathrm{BBO}$ equation, as follow,

$$
\frac{d \vec{U}_{p}}{d t}=\frac{3 C_{D} \rho_{f}}{4 \rho_{p} D_{p}}\left(\vec{U}_{f}-\vec{U}_{p}\right)\left|\vec{U}_{f}-\vec{U}_{p}\right|,
$$

where $U_{p}, \rho_{p}$ and $D_{p}$ are the velocity, density and diameter of a particle, respectively. The drag coefficient $C_{D}$ is expressed as

$$
C_{D}=\left\{\begin{array}{ll}
\frac{24}{\operatorname{Re}_{P}}\left(1+0.15 \operatorname{Re}_{P}^{0.687}\right) & \left(\operatorname{Re}_{P}<1000\right) \\
0.4 & \left(\operatorname{Re}_{P}>1000\right)
\end{array},\right.
$$




$$
\operatorname{Re}_{P}=\frac{D_{P}\left|\vec{U}_{f}-\vec{U}_{p}\right|}{v},
$$

where $\mathrm{Re}_{p}$ is the particle Reynolds number based on the particle diameter and the relative velocity between the gas and the particle. The kinetic viscosity of the gas is denoted by $v$. The particle trajectory is calculated by

$$
\frac{d x_{P}}{d t}=U_{p}
$$

As for the time integration of eqn (4), the leap flog method is employed.

\subsection{Erosion Estimation}

According to the Bitter's work, there are two different mechanisms of sand erosion phenomena $[3,4]$ : deformation and cutting wear. The former is due to the particle velocity normal to the surface and its mass loss is denoted by $W_{D}$ in this paper. The latter is due to the tangential velocity and its mass loss is denoted by $W_{C}$. The total mass loss $W_{T}$ is expressed as a sum of them as,

$$
W_{T}=W_{D}+W_{C}
$$

This Bitter's theoretical erosion model [3, 4], however, is difficult to be used in the simulation for practical applications since the model requires too many parameters. Neilson and Gilchrist $[5,6]$ simplified Bitter model and we employ simplified model. The model equations can be expressed as,

$$
W_{C}=\left\{\begin{array}{cc}
\frac{\frac{1}{2} M(V \sin \alpha-K)^{2}}{\psi}, \\
\frac{1}{\frac{1}{2} M V^{2} \cos ^{2} \alpha \sin n \alpha} \\
\frac{\left(\alpha<\alpha_{0}\right)}{\varphi} & \left(\alpha \geq \alpha_{0}\right) \\
\alpha_{0}=\frac{\pi}{2 n}, &
\end{array}\right.
$$

where $M$ is the mass of a particle, $\alpha$ and $V$ are the attack angle and the impinging velocity of a particle, respectively. $K$ is the threshold value of the velocity component normal to the initial surface. Parameter $n$ is a constant depending on the surface material and $\psi$ and $\phi$ represent the energy required to remove the unit mass of material from the wall by deformation and cutting wear, respectively. According to the experimental study by Neilson and Gilchrist [5, 6], these parameters were confirmed to play an important role for the prediction of sand erosion phenomena.

In the present study, we employ the above-described Neilson-Gilchrist erosion model. However, this model cannot provide the geometrical information of eroded surface (i.e. 


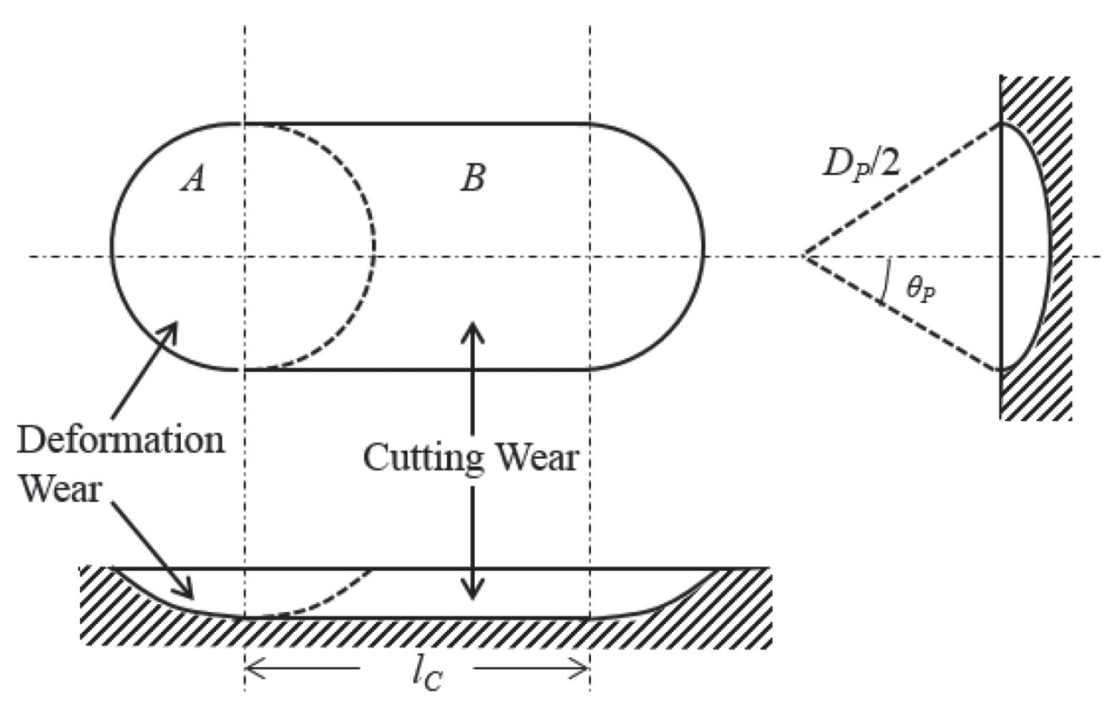

Figure 1: Modelled Erosion Cavity

length and depth of an 'erosion cavity'), because eqns (6) and (7) describe only the mass loss of the surface material. Thus, the geometry of the erosion cavity damaged by a single particle is modelled as shown in Fig. 1: the region 'A' corresponds to the deformation wear; the region ' $\mathrm{B}$ ' corresponds to the cutting wear [8]. The modelled erosion cavity by a single particle is confirmed to be similar to the experimental observation by Bitter [3, 4].

Based on this geometrical assumption, the mass losses due to deformation wear $W_{D}$ and cutting wear $W_{C}$ are,

$$
\begin{gathered}
W_{D}=\rho_{p} \pi\left(\frac{D_{P}}{2}\right)^{3}\left(\frac{\cos ^{3} \theta_{p}+2}{3}-\cos \theta_{p}\right), \\
W_{C}=\rho_{p} l_{C}\left(\frac{D_{P}}{2}\right)^{3}\left(\theta_{p}-\frac{1}{2} \sin 2 \theta_{p}\right) .
\end{gathered}
$$

Erosion length $l_{C}$ is estimated from eqns (9) and (10). We refer this expression as the erosion line approach [8]. The erosion line approach is adopted since the width of the erosion cavity is sufficiently smaller than the three-dimensional grid spacing on the surface. The computational grids consist of two regions, i.e. the flow field and the solid wall. We define the material packing ratio of the solid wall to fluid: when the material packing ratio becomes zero, the grid cell is treated as fluid.

\section{GRID CHARACTERISTICS AND BOUNDARY CONDITIONS}

The computational target is a low-pressure turbine vane cascade using the T106 aerofoil [12]. The simulation model is shown in Fig. 2. We employ a multi-block and an overset method, and defined two kinds of grids, 'O-type sub grid' for the vane and 'H-type flow field main grid' for the flow passage, as shown in Fig. 3. The subgrid includes two regions, flow field and solid wall. Only one stator vane is simulated, under the assumption of geometrical periodicity. The number of grid points in the main grid for the flow field is approximately 220,000. 


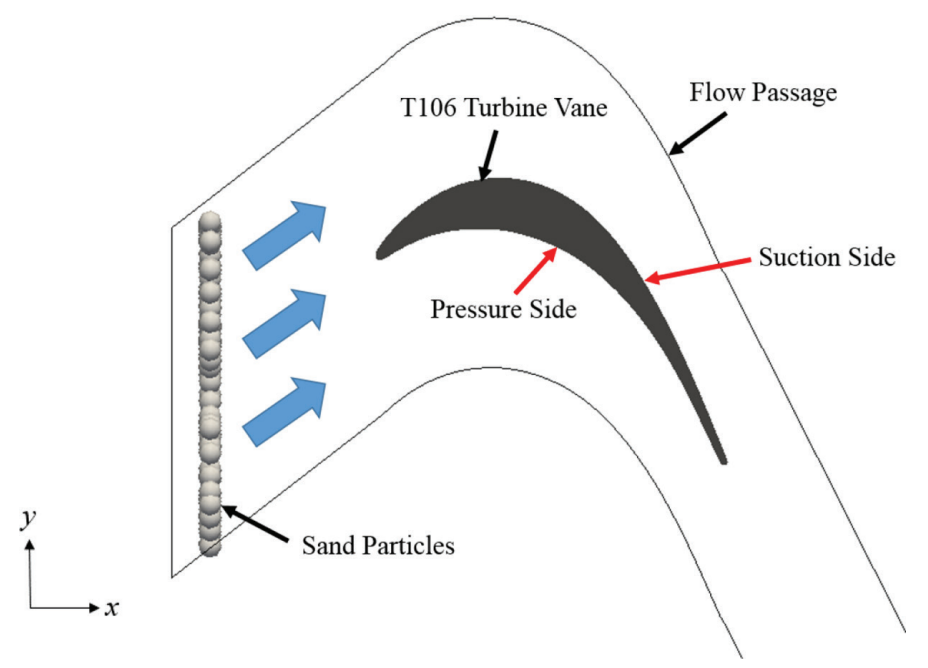

Figure 2: Simulation Model

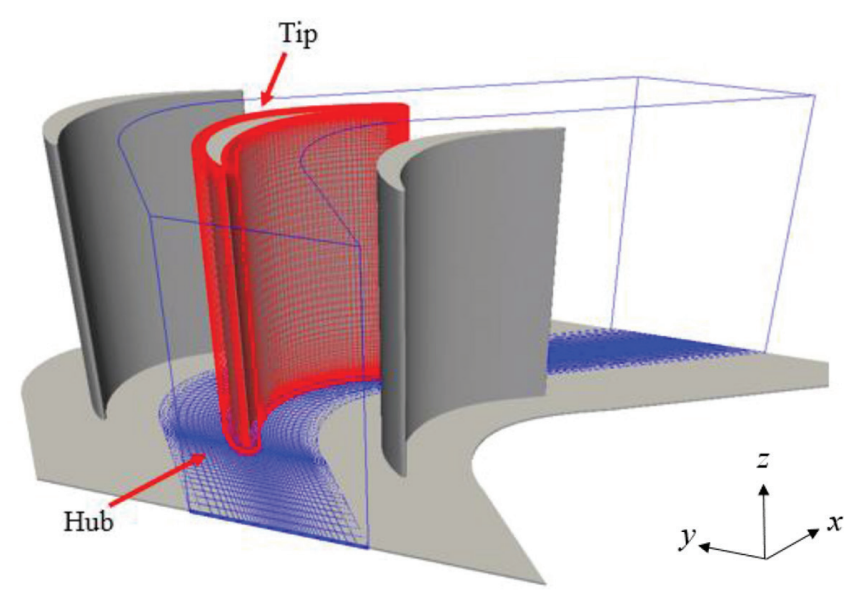

Figure 3: Computational Grid

Those in the sub grid for the flow field and solid wall are approximately 160,000 and 110,000, respectively.

The boundary conditions are imposed as follows. At the inlet boundary, the flow angle, total pressure and total temperature are fixed. For the inflow turbulent kinetic energy, it is assumed that the turbulence intensity is $5.6 \%$. At the outlet, the static pressure is specified. On the vane surface, non-slip and adiabatic conditions are imposed. At the pitchwise and spanwise boundaries, the periodic condition is imposed.We refer to the experimental flow field data from Hoheisel et al. [12]. The material of solid particles is silica, the substrate is made of CMC and the diameter of particles is $50 \mu \mathrm{m}$. These correspond to the experimental conditions [13]. 


\section{RESULTS AND DISCUSSIONS}

Figure 4 shows the instantaneous distributions of Mach number and static temperature. This figure clearly indicates the flow acceleration through the turbine passage and in the vane wake. The inlet Mach number is approximately 0.3 and the exit Mach number is 0.6. Therefore, the flow field is subsonic under this condition.

Figure 5 exhibits the typical trajectories of ten particles around the vane in the cases with and without coating. In the case with coating, erosive particles impinge on the pressure surface and rebound with the high velocity against the vane surface, since the restitution coefficient of the coating is large. Then, solid particle impinges on the suction surface. Some re-collision particles on the pressure surface are also observed. This means that little kinetic energy of the impinging particle is consumed at the impacts, and thus it can be easily expected that severe erosion does not occur in this case. On the other hand, in the case without the coating, erosive particles impinge on the pressure surface, while most of kinetic energy is dissipated by the erosion. The rebound velocity is so low that the rebounded particles impinge again on the pressure surface near the trailing edge. This impingement causes serious erosion near the trailing edge. In the case without coating, the impingement does not occur on the suction surface because the rebounding particles are strongly influenced by the flow field through the turbine passage, since the velocity of the particle is much smaller than the flow. Fig. 6 shows the distribution of the erosion depth on the surface. The depth corresponds to the amount of the erosion. In the case without coating (Figs. 6 (a)-(b)), the erosion depth is very small on the suction side, whereas on the pressure side the erosion depth is uniform in the spanwise direction. The peaky area of the erosion depth is observed around the leading edge and it is around $500 \mu \mathrm{m}$. On the other hand, Figs. 6 (c)-(d) show the coated case. The erosion

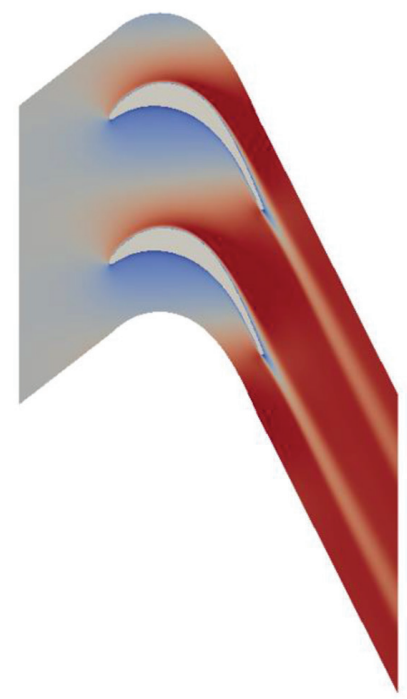

Mach Number

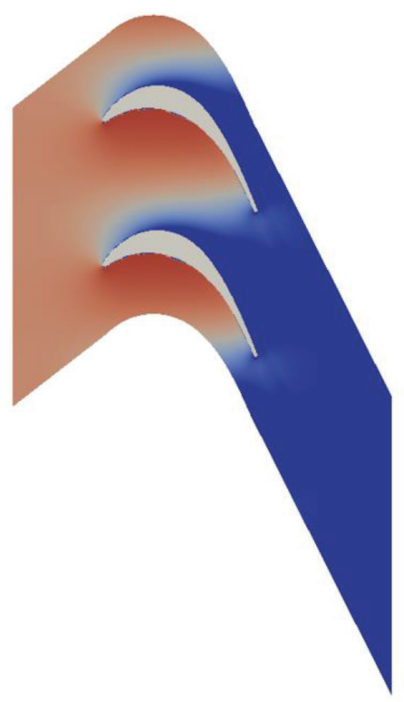

Static Pressure
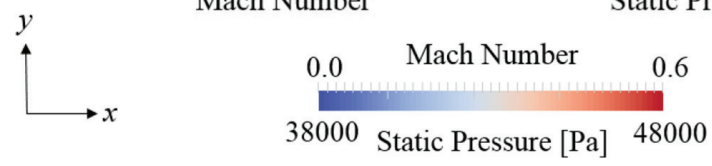

Figure 4: The Distribution of Mach Number and Static Pressure 


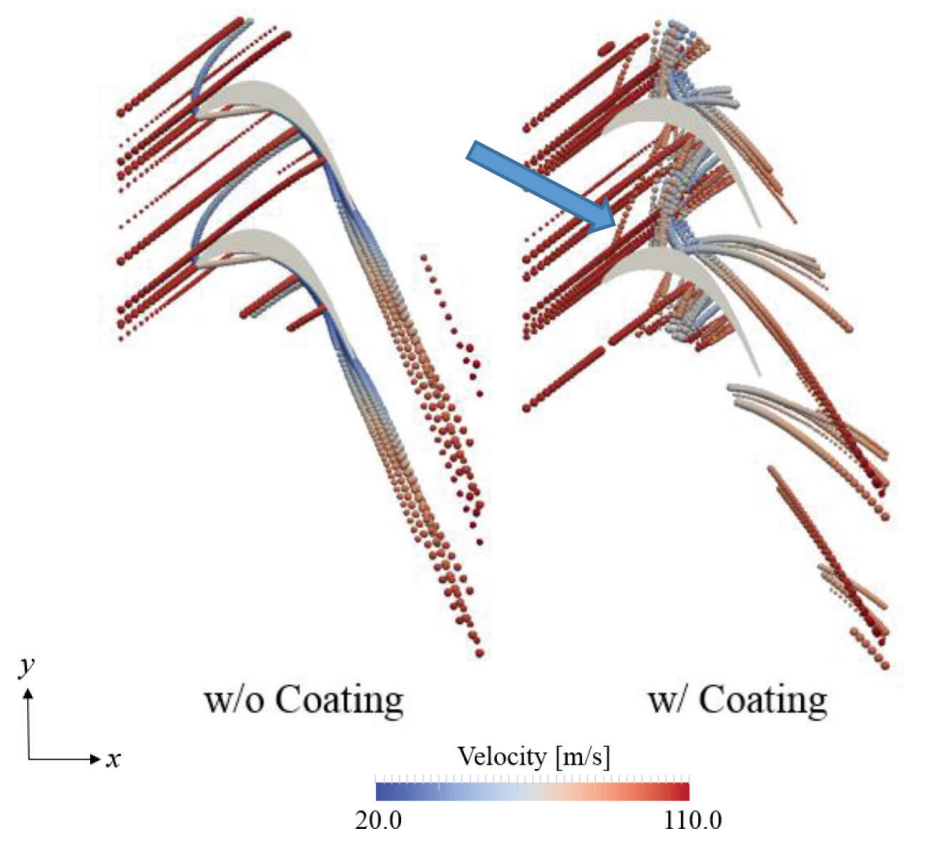

Figure 5: Particle Trajectories

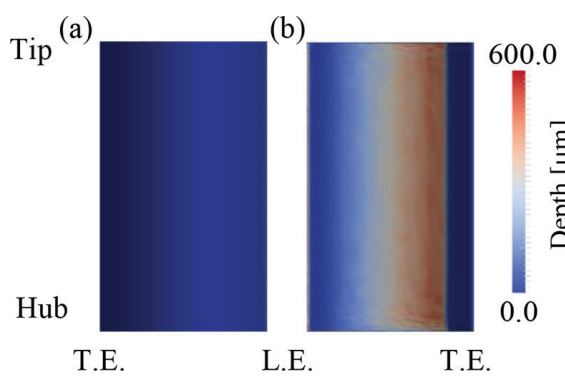

(c)

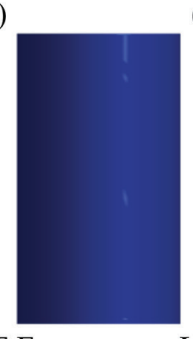

(d)

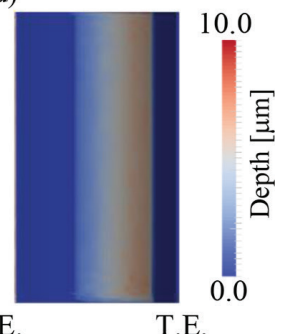

Figure 6: Distributions of the Depth on the Surface: (a), w/o Coating on the Suction Side; (b) w/o Coating on the Pressure Side; (c) w/ Coating on the Suction Side; (d) w/ Coating on the Pressure Side.

depth on the suction side is similar to that on the uncoated case, although the impact frequency of the particles is larger, as mentioned above. It is because the coating plays an important role to protect the vane from sand erosion. Owing to coating, the erosion depth, i.e. the amount of the erosion, on the pressure side also decreases as compared with the uncoated case and the maximum erosion depth is about $6 \mu \mathrm{m}$. Figure 7 shows the impact angle of the particles on the surfaces. The impact angle at the leading edge is about $80 \mathrm{deg}$. in both cases. In the case without coating, the impact angle is very small on the pressure side and its distribution is uniform in the spanwise direction. In the coating case, the high impact angle is found not only in the pressure side, but also in the suction side. In this case, we found that many particles bound with high angle on the pressure side and they impinge again on the suction side. These particles are indicated by a big arrow in Fig. 5. 


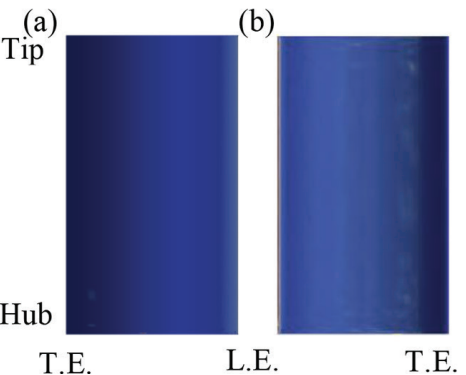

(c)

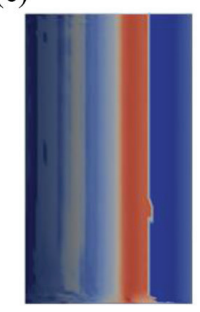

T.E. (d)

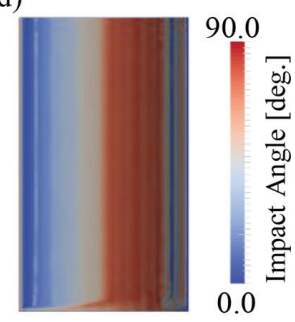

L.E.

T.E.

Figure 7: Distributions of the Impact Angle: (a) w/o Coating on the Suction Side; (b) w/o Coating on the Pressure Side; (c) w/ Coating on the Suction Side; (d) w/ Coating on the Pressure Side.

\section{CONCLUSIONS}

We have carried out the numerical simulations of sand erosion phenomena in the low-pressure turbine vane. We focus on the effect of the coating on the vane. The restitution coefficient affects the erosion patterns on the vane surface. On the suction side of the vane, the particle impact occurs only on the coated vane since the rebound velocity of the particles is very high. On the other hand, the particle impact does not occur on the suction surface of the uncoated vane. In the uncoated case the severe erosion is observed near the leading edge and on the pressure surface. Although the impact frequency of particles is large both on the suction and pressure side in the coated case, the erosion depth is much smaller than that in the uncoated case. From the present results, it is concluded that the coating obviously plays an important role in protecting the $\mathrm{CMC}$ vanes.

\section{ACKNOWLEDGEMENT}

The present study was conducted under the sponsored research by New Energy and Industrial Technology Development Organization (NEDO). We deeply appreciate the financial support.

\section{REFERENCE}

[1] Hamed, A., Tabakoff, W. \& Wenglarz, R., Erosion and deposition in turbomachinery. Journal of Propulsion and Power, 22(2), pp. 350-360, 2006. https://doi.org/10.2514/1.18462

[2] Finnie, I., Erosion of surfaces by solid particles. Wear, 3, pp. 87-103, 1960. https://doi.org/10.1016/0043-1648(60)90055-7

[3] Bitter, J.G.A., A study of erosion phenomena Part I. Wear, 6, pp. 5-21, 1963. https://doi.org/10.1016/0043-1648(63)90003-6

[4] Bitter, J.G.A., A study of erosion phenomena Part II. Wear, 6, pp. 169-190, 1963. https://doi.org/10.1016/0043-1648(63)90073-5

[5] Neilson, J.H. \& Gilchrist, A., Erosion by a stream of solid particle. Wear, 11, pp. 111-122, 1968. https://doi.org/10.1016/0043-1648(68)90591-7

[6] Neilson, J.H. \& Gilchrist, A., An experimental investigation into aspects of erosion in rocket motor nozzles. Wear, 11, pp. 123-143, 1968. https://doi.org/10.1016/0043-1648(68)90592-9 
[7] Balan, C. \& Tabakoof, W., Axial flow compressor performance deterioration, AIAA84-1208, 1984.

https://doi.org/10.2514/6.1984-1208

[8] Suzuki, M., Inaba, K. \& Yamamoto, M., Numerical simulation of sand erosion phenomena in rotor/stator interaction of compressor. The Japan Society of Mechanical Engineers, 76(09-0933), pp. 765-803, 2008.

[9] Kato, M. \& Launder, B.E., The modeling of turbulent flow around stationary and vibrating square cylinder. Proceedings, 8th Symposium on Turbulent Shear Flows, 10-4-1-104-6, 1993.

[10] Yee, H.C., Upwind and symmetric shock capturing schemes, NASA-TM-89464, 1987.

[11] Fujii, K. \& Obayashi, S., Practical application of improved LU-ADI scheme for the three-dimensional Navier-Stokes computations of transonic viscous flows, AIAA Paper, 86-0513, 1987.

[12] Hoheisel, H., Test cases for computation of internal flows in aero engine components. AGARD-AR-275, pp. 112-123, 1990.

[13] Suzuki, M. et al. (Private Communication, 2015). 